\title{
Determinants of symptom interval in childhood
}

\section{cancer}

\author{
Vaskar Saha, Sharon Love, Tim Eden, Paul Micallef-Eynaud, Gordon MacKinlay
}

\begin{abstract}
The duration of symptoms before diagnosis (lag time) was defined for 184 of 236 children diagnosed as having a malignancy at the Royal Hospital for Sick Children, Edinburgh for the time period January 1982 until December 1990. The natural logarithm of the lag time was correlated with age, gender, diagnostic group, white cell count in acute leukaemia, clinical stage of disease in solid tumours, and event free survival. Age was significantly associated with lag time, older children presenting later. In the diagnostic groups, mean lag time ranged from 2.8 weeks in nephroblastoma to 13.3 weeks for brain tumours. Diagnostic group was predictive for lag time after adjustment for age, with for example, a significantly longer lag time for those with brain tumours. However lag time was not predictive of event free survival and it is likely that lag time has other major determinants. When compared with previous studies, there also appears to be a regional variation in lag time for diagnostic groups. It seems likely that this is a reflection of geographical difference in the structure of health systems and is therefore yet another important determinant.

(Arch Dis Child 1993; 68: 771-774)
\end{abstract}

The symptoms and signs of cancer in children are caused by the effect of tumour mass on surrounding normal structures, secretion by the malignancy of a substance that disturbs normal function, or bone marrow failure. As a result, the commonest presenting features are fever, headache, vomiting, pallor and fatigue, bone pain, limping, weight loss, bleeding and/or the presence of a mass. Unfortunately, many of these symptoms and signs commonly occur in less sinister childhood illnesses. Justifiably, a diagnosis of malignancy is often not immediately considered when a child first presents and investigations for more benign disorders are initiated. As a consequence, most children with cancer are symptomatic for a period of time before the diagnosis is made. This period has been termed the lag time.

To date, only three studies have examined factors that influence the lag time in childhood cancer. Pratt et al analysed the presenting symptoms in 109 cases of rhabdomyosarcoma. ${ }^{2}$ Flores et al found that the lag time for brain tumours was significantly longer than that for nephroblastomas or acute leukaemia. ${ }^{3}$ The most recent and complete study by Pollock et al found that the lag time was strongly associated with age and tumour type in childhood solid tumours. In their study. Ewing's sarcoma not brain tumours was the malignancy with the longest lag time. ${ }^{4}$ All the authors have suggested that a shorter lag time could improve the prognosis and have recommended that primary care physicians maintain a high index of suspicion. Certainly, parents frequently tend to blame the primary care doctor for a 'delay in the diagnosis'. Questions are also raised in the minds of the medical team as to whether an earlier referral could have been made and if so did the 'delay' adversely affect the prognosis

Pollock et al could only attribute $16 \%$ of the observed variance in lag time to the age at presentation and diagnosis. ${ }^{4}$ It is quite likely then that the lag time is the outcome of a number of determinants. Factors such as parental attention, physician quality, and health care structures are all important but difficult to assess directly. All three previous studies are from different areas of the US. Though the characteristics of disease are well recognised, health care systems show considerable geographical difference. ${ }^{5}$ If the regional health structure contributes significantly to the lag time we would expect to find geographical variations in the already established determinants of what delays diagnosis. This study was designed therefore to examine first the relation of lag time within multiple diagnostic groups and with age, gender, and extent of disease in a cohort from the UK and then compare the results with those of the previous studies.

\section{Patients and methods}

We carried out a retrospective analysis of all children (aged 0-15 years) diagnosed as having cancer at the Royal Hospital for Sick Children, Edinburgh during the period of January 1982 through December 1990. A child was considered to be symptomatic from the day that unrelieved symptoms that could be directly attributed to a malignancy were first recorded. The lag time was calculated from the date of onset of symptoms until the date of diagnosis to the nearest week.

The natural logarithm of lag time (used as lag time had a skewed distribution ${ }^{4}$ ) was then correlated with the gender, age at presentation, tumour type, clinical stage of disease in solid tumours where possible, and presenting white cell count in acute leukaemia using a one way analysis of variance. Those variables found to be significant at the $5 \%$ level or less were included in a multiway analysis of variance. Results and significance of the $F$ test have been presented. The mean lag time in the diagnostic groups was compared with those obtained in the previous studies. ${ }^{2-4}$ Cox regression analysis was used to assess if lag time was independently predictive of event free survival, ${ }^{6}$ having adjusted for other determinants. 
Table 1 Patient characteristics and lag time in weeks

\begin{tabular}{|c|c|c|c|}
\hline \multirow[b]{2}{*}{ Patient characteristic } & \multirow{2}{*}{$\begin{array}{l}\text { No of } \\
\text { patients }\end{array}$} & \multicolumn{2}{|c|}{ Lag time (weeks) } \\
\hline & & $\operatorname{Mean}(S D)$ & Median (range) \\
\hline Male & 101 & $7 \cdot 3(8 \cdot 3)$ & $4(1-52)$ \\
\hline Female & 83 & $7 \cdot 1(10 \cdot 3)$ & $4(1-72)$ \\
\hline \multicolumn{4}{|l|}{ Age at diagnosis (years) } \\
\hline $0-1$ & 31 & $5 \cdot 5(9 \cdot 8)$ & $3(1-52)$ \\
\hline $2-3$ & 46 & $4 \cdot 8(6 \cdot 3)$ & $3(1-36)$ \\
\hline $4-5$ & 28 & $6 \cdot 2(5 \cdot 4)$ & $4(1-24)$ \\
\hline $6-7$ & 28 & $6 \cdot 6(6 \cdot 2)$ & $4(1-20)$ \\
\hline $8-9$ & 17 & $8 \cdot 6(6 \cdot 8)$ & $6(2-24)$ \\
\hline $10-11$ & 21 & $12 \cdot 2(16 \cdot 1)$ & $6(1-72)$ \\
\hline$\geq 12$ & 13 & $12 \cdot 8(11 \cdot 6)$ & $8(1-38)$ \\
\hline \multicolumn{4}{|l|}{ Diagnosis ${ }^{\star}$} \\
\hline Acute leukaemia & 65 & $5 \cdot 0(5 \cdot 0)$ & $3(1-32)$ \\
\hline Brain tumour & 28 & $13 \cdot 3(14 \cdot 7)$ & $7(1-72)$ \\
\hline Bone tumour & 12 & $8 \cdot 4(6 \cdot 7)$ & $8(1-24)$ \\
\hline Lymphoma & 17 & $8 \cdot 4(9 \cdot 0)$ & $6(1-38)$ \\
\hline Neuroblastoma & 8 & $5 \cdot 4(7 \cdot 7)$ & $2(1-24)$ \\
\hline Rhabdomyosarcoma & 20 & $6 \cdot 7(7 \cdot 4)$ & $4(1-24)$ \\
\hline Nephroblastoma & 18 & $2 \cdot 8(2 \cdot 5)$ & $1(1-12)$ \\
\hline \multicolumn{4}{|c|}{ White cell count $\left(\times 10^{9} / 1\right) \dagger$} \\
\hline$\geq 50$ & 12 & $3.9(2 \cdot 7)$ & $3(1-12)$ \\
\hline$<50$ & 53 & $5 \cdot 3(5 \cdot 3)$ & $3 \cdot 5(1-32)$ \\
\hline \multicolumn{4}{|l|}{ Stage $\ddagger$} \\
\hline I & 11 & $4 \cdot 8(6 \cdot 9)$ & $1(1-24)$ \\
\hline II & 13 & $5 \cdot 9(4 \cdot 8)$ & $6 \cdot 5(1-20)$ \\
\hline III & 16 & $8.8(10.4)$ & $4(1-38)$ \\
\hline IV and V & 24 & $4 \cdot 2(4 \cdot 2)$ & $3(1-20)$ \\
\hline
\end{tabular}

* Sixteen patients were diagnosed to have malignancies other than these.

In those children with acute leukaemia

†Clinical staging in 64 children with solid tumours.

Table 2 Comparison of lag times between other diagnostic groups and acute leukaemia

\begin{tabular}{lclrc}
\hline Diagnostic group & Coefficient $^{\star}$ & \multicolumn{1}{l}{$S E$} & \multicolumn{1}{l}{$t$} & \multicolumn{1}{c}{$p$} \\
\hline Brain tumour & 0.71 & 0.17 & 4.29 & $<0.001$ \\
Bone tumour & 0.44 & 0.23 & 1.89 & 0.06 \\
Lymphoma & 0.35 & 0.20 & 1.74 & 0.08 \\
Neuroblastoma & -0.08 & 0.28 & -0.30 & 0.8 \\
Rhabdomyosarcoma & 0.11 & 0.19 & 0.57 & 0.6 \\
Nephroblastoma & -0.40 & 0.20 & -2.06 & 0.04
\end{tabular}

*The coefficients are the difference in logarithm of lag time between, and the $t$ tests consider, each diagnostic group individually with the acute leukaemia diagnostic group.

Table 3 Comparison of lag times between other diagnostic groups and acute leukaemia adjusting for age

\begin{tabular}{lclcc}
\hline Diagnostic group & Coefficient $^{\star}$ & $S E$ & \multicolumn{1}{c}{$t$} & \multicolumn{1}{c}{$p$} \\
\hline Brain tumour & 0.67 & 0.16 & 4.23 & $<0.001$ \\
Bone tumour & 0.17 & 0.23 & 0.72 & 0.5 \\
Lymphoma & 0.16 & 0.20 & 0.79 & 0.4 \\
Neuroblastoma & 0.19 & 0.27 & 0.71 & 0.5 \\
Rhabdomyosarcoma & 0.09 & 0.18 & 0.50 & 0.6 \\
Nephroblastoma & -0.32 & 0.19 & -1.74 & 0.08
\end{tabular}

*The coefficients are the difference in logarithm of lag time between, and the $t$ tests consider, each diagnostic group individually with the acute leukaemia diagnostic group.
Table 4 Mean lag times in the different diagnostic groups in this and previous studies

\begin{tabular}{|c|c|c|c|c|}
\hline \multirow[b]{2}{*}{ Diagnosis } & \multicolumn{4}{|c|}{ Lag time (weeks) } \\
\hline & $\begin{array}{l}\text { Saha et al } \\
\text { (Edinburgh) }\end{array}$ & $\begin{array}{l}\text { Pratt et al }{ }^{2} \\
\text { (Memphis) }\end{array}$ & $\begin{array}{l}\text { Flores et al }{ }^{3} \\
(\text { Atlanta })\end{array}$ & $\begin{array}{l}\text { Pollock et al }{ }^{+} \\
\text {(St Louis) }\end{array}$ \\
\hline $\begin{array}{l}\text { Acute leukaemia } \\
\text { Brain tumour } \\
\text { Nephroblastoma } \\
\text { Bone tumour } \\
\text { Lymphoma } \\
\text { Rhabdomyosarcoma } \\
\text { Neuroblastoma }\end{array}$ & $\begin{array}{c}5 \cdot 4 \\
13 \\
2 \cdot 5 \\
8 \cdot 4 \\
8 \cdot 4 \\
6 \cdot 6 \\
5 \cdot 3\end{array}$ & $\begin{array}{l}\text { NA } \\
\text { NA } \\
\text { NA } \\
\text { NA } \\
\text { NA } \\
6-8(109) \\
\text { NA }\end{array}$ & $\begin{array}{l}4 \cdot 5(123) \\
26(79) \\
2 \cdot 8(45) \\
\text { NA } \\
\text { NA } \\
\text { NA } \\
\text { NA }\end{array}$ & $\begin{array}{l}\text { NA } \\
9 \cdot 4(380) \\
\text { NA } \\
11 \cdot 5-20 \cdot 8(528) \\
7 \cdot 1-14(897) \\
\text { NA } \\
5 \cdot 4(860)\end{array}$ \\
\hline
\end{tabular}

$\mathrm{NA}=$ diagnostic group not analysed in this study

Figures in parentheses denote the number in each diagnostic group.

\section{Results}

Of the 236 children diagnosed during this period, it was possible to date the onset of symptoms accurately in $184(78 \%)$. The patient characteristics are shown in table 1 , though age was not grouped for analysis. There was no significant difference in the lag time between males and females with an estimated ratio of lag time, female to male, of $1 \cdot 2$ (95\% confidence interval (CI) of 0.9 to $1 \cdot 6, p=0.22)$. Age was a significant predictor for lag time, $F(1,182)=$ $24 \cdot 1, \mathrm{p}<0.001$, with older children having a longer lag time.

As shown in table 1, 168 patients were diagnosed as having either acute leukaemia, a brain tumour, bone tumour, lymphoma, neuroblastoma, rhabdomyosarcoma, or nephroblastoma. Of the remaining 16, three had chronic myeloid leukaemia and the rest a variety of other solid tumours. These 16 patients are omitted from any analysis using diagnostic groups. The mean lag time varied from $2 \cdot 8$ weeks for nephroblastoma to 13.3 weeks in brain tumour. One way analysis of variance showed diagnostic group to be significant for length of lag time, $\mathrm{F}(6,161)=5 \cdot 5, \mathrm{p}<0 \cdot 001$. The results for each diagnostic group compared with the acute leukaemia group are shown in table 2 . The lag time for brain tumours was significantly longer than for acute leukaemia, the estimated ratio of lag time of brain tumours to acute leukaemia was $2.0(95 \% \mathrm{CI}$ of 1.5 to $2 \cdot 8, \mathrm{p}=$ $<0.001$ ), that is children with brain tumours were estimated to have twice the lag time of children with leukaemia. Bone tumour, lymphoma, and nephroblastoma were of a borderline significant difference when compared with acute leukaemia, with the former two having a longer lag time and nephroblastoma having a shorter lag time.

As both age and diagnostic group were shown by univariate analysis of variance to be predictive for lag time, these were put into a multiway analysis of variance. Both age, $\mathrm{F}(1,160)=16.96$, $\mathrm{p}<0.001$ and diagnostic group, $\mathrm{F}(6,160)=4.41$, $\mathrm{p}<0.001$ were individually significant and the interaction terms were not significant. Table 3 shows the comparison between the other diagnostic groups and acute leukaemia with adjustment for age. Diagnostic group remains predictive with this adjustment for age, with the ratio between brain tumour and leukaemia estimated to be 2.0 (95\% CI of 1.4 to 2.7 , $\mathrm{p}=<0.001$ ).

Of the 65 children with acute leukaemia, 12 presented with a white cell count of $\geq 50 \times 10^{9} / 1$. As shown in table 1 , the mean lag time in this group was 3.9 weeks compared with 5.3 in the 53 children with a lower white cell count. This difference was not significant; the estimated ratio of lag times in those with a presenting white cell count of $\geq 50 \times 10^{9} / 1$ as compared with those with a count $<50 \times 10^{9} / 1$ was $1 \cdot 1(95 \%$ CI of 0.6 to 1.4 and $\mathrm{p}=0 \cdot 7$ ).

Clinical staging systems are different for each solid tumour and some tumours, for example brain tumours, are not usually staged. However, an advanced stage is indicative of advanced disease in all diagnostic groups. In 64 children with a solid tumour clinical staging of 
disease at presentation was possible. The mean lag times for those diagnosed to be in stages I, II, III, IV, and V are shown in table 1. The difference in lag time between the stages is not significant, $F(3,60)=1 \cdot 46, p=0 \cdot 23$.

The logarithm of lag time was put with diagnosis, sex, and age into a forward stepwise Cox regression model for event free survival. Only diagnosis was significantly predictive of event free survival; children with a diagnosis of rhabdomyosarcoma had a significantly shorter event free survival period than children with other malignancies (coefficient $=0 \cdot 8787, \mathrm{SE}=$ $0.3118, p=0.005$ ). Forcing the logarithm of lag time into this model lag time was not predictive of event free survival (coefficient $=0.0221, \mathrm{SE}=$ $0 \cdot 1321, \mathrm{p}=0.8)$. Using acute leukaemia patients only (as this was the largest group) sex, age, and logarithm of lag time were not significantly predictive of event free survival ( $p>0.05)$.

Table 4 shows the mean lag time in the diagnostic groups from the various studies. The mean lag time of rhabdomyosarcoma in our study is similar to that seen by Pratt et al. ${ }^{2}$ Flores et al also found that brain tumours had the longest lag time. ${ }^{3}$ While the lag time for acute leukaemia and nephroblastoma are comparable in our study with that of Flores et al, they described a mean lag time for brain tumours of 26 weeks while in our study it was 13 weeks. On the other hand, Pollock et al found the lag time for brain tumours to be 9.4 weeks. ${ }^{4}$ The longest lag time in their study was seen in children with bone tumours, mean lag time in those with osteosarcoma being 11.5 weeks and those with Ewing's sarcoma 20.8 weeks. The lag times in children with lymphoma $(7 \cdot 1$ weeks in nonHodgkin's lymphoma and 14 weeks in Hodgkin's disease) and neuroblastoma are comparable.

\section{Discussion}

In this study, lag time was shown to be significantly correlated with the age at presentation and the actual diagnosis. Multiway analysis of variance showed that age and diagnosis are independently associated with lag time and after adjustment for age the diagnosis continues to have a statistically significant association with the lag time. These findings are in agreement with the one previous study which similarly analysed factors that affect lag time. ${ }^{3}$ In the UK and USA younger children are likely to be seen more often by a physician than older children or adolescents and this may tend to decrease the lag time. ${ }^{4}$ Additionally, detection of symptomatic disease in older children is dependent greatly on self reporting and may be particularly unreliable for adolescents. Therefore increased parent and physician awareness of a child's condition may account for a shorter lag time in younger children. ${ }^{4}$ In addition, the presenting features are a result of the effect of malignant tissue on surrounding structures. In younger children, reduced organ volume may also lead to a more rapid progression of symptoms with shortening of the lag time. This study and the previous ones $^{34}$ are in agreement that the diagnostic group is predictive of the lag time. However, while in this analysis and that of Flores et al brain tumours had a longer lag time, ${ }^{3}$ Pollock et al found bone tumours and in particular Ewing's tumour to have the most significantly prolonged lag times. ${ }^{4}$ Additionally, while the lag times in some of the diagnostic groups were similar, they were markedly different in others and this included the group with brain tumours.

Brain tumours vary from benign to extremely malignant. It may be argued therefore that the variation in lag time in the three studies reflects the differences in the brain tumour populations. Flores et al also found that site of the brain tumour, not the histological diagnosis, appeared to influence lag time. ${ }^{3}$ In their series mean lag time in supratentorial $(38 \%)$ and infratentorial $(62 \%)$ tumours was 43.4 weeks and 10.8 weeks respectively. While no such information was available from Pollock et al, the majority (75\%) of the study population had infratentorial neoplasms. In other words, the populations are not dissimilar. It is likely therefore, that the variation in lag times seen in the diagnostic groups is due to geographical variations in health care systems, for example ease of access to medical care, presence of specialised paediatric oncology units, etc. ${ }^{78}$

Lag time could be influenced by the rate at which the tumour enlarges or spreads. If so, rapidly progressive disease, that is malignancies that are in an advanced stage at the time of diagnosis, would have a shorter lag time. However, in this study we found no correlation between the lag time and the stage of solid tumours or the presenting white cell count in acute leukaemia.

For a paediatric oncologist, the index of suspicion of cancer is very high. The opposite is true for the general physician. If anything, there may be a general reluctance to consider such a diagnosis as cancer carries with it the fear of death, is rare in childhood, and is an unfamiliar diagnosis for the non-specialist. ${ }^{9}$ It has been argued that increased vigilance on the part of the physician could lead to a shortening in the lag time. ${ }^{2-4}$ Logically, if the lag time is a function of delay in diagnosis rather than in the nature of the disease, a shorter lag time should improve prognosis. In this study we have been unable to find a positive correlation between length of lag time and outcome. This suggests that it is the nature and epidemiology of disease that are the important determinants for lag time. Therefore, we do not feel that the onus should be on the primary care physician to make an early diagnosis. Except in rare, individual cases $^{310}$ it is more likely that the role of the general physician does not significantly affect lag time. Instead it is a subsidiary factor that contributes to the health care structure, itself a significant determinant.

This study has obvious limitations. The numbers in each diagnostic category are small. As analysis relied on retrospective review of medical records, it was not possible to assess the reliability with which the date of symptom onset was recorded. Nor has it been possible to analyse directly the role of physician, parent, or health care systems. However, the results of this study indicate that age at presentation and actual diagnosis are determinants for lag time, that 
there is a geographical variation in lag time in the diagnostic groups for which the most probable explanation is the variation in health support systems, and that lag time is not related to survival.

The authors would like to thank Dr Ian Hann and Miss Rebecca Brown for their advice during the preparation of this manuscript. VS is a recipient of a Leonora Knatchbull Paediatric Oncology Fellowship.

1 Worden JW, Weisman AD. Psychosocial components of la ime in cancer diagnosis. F Psychosom Res 1975; 19: 69-79.

2 Pratt CB, Smith JW, Woerner S, et al. Factors leading to delay in the diagnosis and affecting survival of children with head neck rhabdomyosarcoma. Pediatrics 1978; 61: 30-4.

3 Flores LE, Williams DL, Bell BA, O'Brien M, Ragab A.
Delay in the diagnosis of pediatric brain tumours. Am 7 Dis Child 1986; 140: 684-6.

4 Pollock BH, Krischer JP, Vietti TJ. Interval between symptom onset and diagnosis of pediatric solid tumours.

5 Brook RH, Lohr KN, Chassin MR, Kosecoff J, Fink A, Solomon D. Geographic variations in the use of services: do they have any clinical significance? Health Aff (Millwood) 1984; 3: 63-73.

6 Cox DR. Regression models and life tables. Fournal of the Royal Statistical Society 1972; 34: 187-202.

7 Chassin MR, Brook RH, Park RE, et al. Variations in the use of medical and surgical services in the medical population. of medical and surgical services

8 Chassin MR, Kosecoff J, Winslow CM, et al. Does inappropriate use explain geographic variations in the use of health priate use explain geographic variation
services? FAMA 1987; 258: 2533-7.

9 Nesbit ME Jr. Clinical assessment and differential diagnosis of the child with suspected cancer. In: Pizzo PA, Poplack DG, ed. Principles and practice of pediatric oncology. Philadelphia: JB Lippincott, 1989: 83-92.

10 Villani R, Gaini SN, Tomei G. Follow-up study of brain tumours in children. Childs Brain 1975; 1: 126-35. 